\title{
Apomorphine-Induced Prepulse Inhibition Disruption is Associated with a Paradoxical Enhancement of Prepulse Stimulus Reactivity
}

\author{
Benjamin K Yee*,', Holger Russig' and Joram Feldon' \\ 'Laboratory of Behavioural Neurobiology, Swiss Federal Institute of Technology, Zurich, Schwerzenbach, Switzerland
}

\begin{abstract}
Prepulse inhibition (PPI) refers to the reduction in startle reaction to a startle-eliciting stimulus when it is shortly preceded by a subthreshold prepulse stimulus. PPI has been extensively employed as an assay for sensorimotor gating, and its disruption has been characterized in specific disease conditions, including schizophrenia. In animals, dopamine agonists disrupt PPI, and this disruption can be antagonized by antipsychotic drug treatment. The present study extended these fundamental findings to C57BL6 mice, and further evaluated the subjects' reaction to the prepulse stimulus alone in relation to the expression of PPI. Not only did apomorphine $(2.0 \mathrm{mg} / \mathrm{kg}$, intraperitoneal (i.p.)) attenuate PPI but it also enhanced reactivity to the prepulse stimulus. The dual effects of apomorphine appear paradoxical in view of the positive correlation, detectable in both the control and apomorphine groups, between prepulse reactivity and PPI magnitude. The present findings contradict the hypothesis that apomorphine disrupts PPI via reduced detectability or perception of the prepulse, and we further propose that enhanced distractibility may provide a parsimonious account for the dual effects of apomorphine. Moreover, haloperidol pretreatment $(0.4 \mathrm{mg} / \mathrm{kg}$, i.p.) fully antagonized the effects of apomorphine upon prepulse reactivity as well as on PPI. The present results add to our understanding of the relevance and applicability of the PPI paradigm in modeling schizophrenia-like symptoms in animals.

Neuropsychopharmacology (2004) 29, 240-248, advance online publication, 3 December 2003; doi: I 0.1038/sj.npp. 1300323
\end{abstract}

Keywords: amphetamine; apomorphine; haloperidol; mice; prepulse inhibition; schizophrenia; startle

\section{INTRODUCTION}

Prepulse inhibition (PPI) refers to the reduction of startle reaction to a startle-eliciting stimulus when it is shortly preceded by a weak stimulus (Hoffman and Searle, 1965). PPI of the acoustic startle reflex has been extensively studied in animals and in humans. Theoretical expositions of PPI invariably attribute it to a competition between the prepulse and pulse stimuli for limited processing resources. When the prepulse and pulse stimuli are presented in close proximity, the preceding prepulse triggers a protective or gating mechanism that limits the processing of, and therefore the reaction to, the succeeding pulse stimulus (Braff et al, 1992; Graham, 1975, 1980, 1992).

As reviewed elsewhere, significant deficits in PPI have been reported in schizophrenic, schizotypal, and presum-

\footnotetext{
* Correspondence: BK Yee, Laboratory of Behavioural Neurobiology, Swiss Federal Institute of Technology, Zurich, Schorenstrasse 16, Schwerzenbach 8603, Switzerland, Tel: +4I I 6557203, Fax: +4I I 6557203, E-mail: benjamin.yee@behav.biol.ethz.ch

Received 22 May 2003; revised 01 September 2003; accepted 09 September 2003

Online publication: 10 September 2003 at http://www.acnp.org/ citations/Npp09100303233/default.pdf
}

ably psychosis-prone subjects (Braff et al, 1992, 2001). Although PPI impairment is not unique to schizophrenia (Braff et al, 2001; Castellanos et al, 1996; Swerdlow et al, 1995), the sensitivity of PPI to dopaminergic manipulations fits readily to the dopamine hypothesis of schizophrenia (Snyder, 1976). Systemic administration of the direct dopamine agonist, apomorphine, reliably disrupts PPI, and such disruption is antagonized by antipsychotic drugs (Swerdlow et al, 1994). Apomorphine-induced PPI disruption has therefore been commonly employed as a screening test for potential antipsychotic compounds (Swerdlow and Geyer, 1998). However, the psychological mechanism whereby apomorphine leads to PPI disruption remains a subject of speculation.

One suggestion contends that apomorphine attenuates the PPI effect by reducing the detectability of the weak prepulse stimulus (Davis et al, 1990). Alternatively, apomorphine may directly interfere with the gating mechanism underlying PPI. Although the two possibilities are not mutually exclusive, they could be distinguished experimentally. One way is to evaluate the drug's effect on the processing of the prepulse stimulus. If apomorphine impairs the perception and/or processing of the prepulse, then the reactivity towards the prepulse should be 
diminished. On the other hand, if apomorphine solely impairs the gating mechanism, no such change in prepulse perception would be expected (cf Braff et al, 1992).

The present study examined the possible relationships that might exist between prepulse reactivity and the expression of PPI in C57BL/6J mice under different pharmacological interventions. To this end, our experimental design included trials in which only the prepulse stimulus was presented. Such trials are not always present in existing PPI studies, and even if they are included they are seldom subjected to vigorous analysis. The major focus here is to examine whether animals that reacted more strongly to the prepulse also tended to express stronger PPI. In most studies, prepulses of various intensities are usually employed, and it is a well-established finding that more intense prepulses lead to stronger PPI. The present study, on the other hand, examined this relationship in terms of individual differences among animals. A positive relationship between reactivity to prepulse and PPI magnitude would be expected based on the theoretical accounts put forward by Graham $(1975,1980,1992)$ and Braff et al (1992). Experiments 1 and 2 examined the effects of apomorphine and amphetamine, respectively, upon prepulse reactivity, PPI magnitude, as well as the relationship between them. Experiment 1 would constitute a direct test of the hypothesis of Davis et al (1990) that apomorphine attenuates PPI via reduced detectability of the prepulse. Experiment 3 was designed to further examine whether the ability of haloperidol to counter the disruptive effect of apomorphine upon PPI was associated with any effects on the prepulse reactivity or detection. One extension of the suggestion by Davis et al (1990) is that antipsychotic drugs (such as haloperidol) might lead to enhanced reaction or detection of the prepulse stimulus, which would also predict the finding that antipsychotic drugs are associated with enhanced PPI when administered on their own (Geyer et al, 2001).

\section{METHODS}

\section{Subjects}

Three cohorts of naïve male C57BL6/J mice, weighing 28$35 \mathrm{~g}$, were used. Littermates of three to four mice were kept together in a cage, and maintained under ad libitum food and water throughout the experiment. They were housed in a temperature- and humidity-controlled $\left(22^{\circ} \mathrm{C}, \sim 55 \%\right)$ vivarium under a reversed light-dark cycle (lights off: 08002000). All experiments were conducted in the dark phase of the cycle. In each experiment, littermates from each litter were distributed to different treatment groups as far as possible in order to minimize the potential confounds resulting from litter effects (Zorrilla, 1997).

\section{Drugs}

Apomorphine $\mathrm{HCl}$ and $\mathrm{D}$-amphetamine sulfate (obtained from Sigma Chemicals, St Louis, USA) were dissolved in $0.1 \%$ ascorbic acid (vitamin C, VitC) and $0.9 \% \mathrm{NaCl}$ solution, respectively, to achieve the desired concentration for injection. Haloperidol was obtained from Janssen-Cilag (Baar, Switzerland), in the form of ampoules consisting of
$5 \mathrm{mg}$ of haloperidol in $1 \mathrm{ml}$ of solvent containing $6 \mathrm{mg}$ of lactic acid. This was diluted with sterile $0.9 \% \mathrm{NaCl}$ solution to achieve the required concentration (final $\mathrm{pH}=5.5$ ). The appropriate vehicle solution was used for the control group: $0.1 \%$ ascorbic acid for apomorphine, $0.9 \% \mathrm{NaCl}$ for amphetamine, and $0.9 \%$ saline/lactic acid $(\mathrm{pH} 5.5)$ for haloperidol. D-amphetamine $(2.5 \mathrm{mg} / \mathrm{kg})$ and haloperidol $(0.4 \mathrm{mg} / \mathrm{kg})$ were administered via the intraperitoneal (i.p.) route, while apomorphine $(2.0 \mathrm{mg} / \mathrm{kg})$ was administered via the subcutaneous (s.c.) route. Haloperidol was administered $60 \mathrm{~min}$ prior to testing, and amphetamine and apomorphine were administered $15 \mathrm{~min}$ before testing. The volume of injection was $5 \mathrm{ml} / \mathrm{kg}$. The doses and pretreatment time were determined by previous pilot studies and conformed to those commonly employed in studies of a similar nature in mice (for a review, see Geyer et al, 2002).

\section{Apparatus}

The apparatus consisted of two acoustic startle chambers for mice (SR-LAB, San Diego Instruments, San Diego, CA, USA). Each startle chamber comprised a nonrestrictive cylindrical enclosure made of clear Plexiglas attached horizontally on a mobile platform, which was in turn resting on a solid base inside a sound-attenuated isolation cubicle. A high-frequency loudspeaker mounted directly above the animal enclosure inside each cubicle produced a continuous background noise of $65 \mathrm{~dB}_{\mathrm{A}}$ and the various acoustic stimuli in the form of white noise. Vibrations of the Plexiglas enclosure caused by the whole-body startle response of the animal were converted into analog signals by a piezoelectric unit attached to the platform. These signals were digitized and stored by a computer. A total of 130 readings were taken at $0.5-\mathrm{ms}$ intervals (ie spanning across $65 \mathrm{~ms}$ ), starting at the onset of the startle stimulus in pulse-alone and prepulse-plus-pulse trials, and at the onset of the prepulse stimulus in prepulse-alone trials. The average amplitude over the $65 \mathrm{~ms}$ was used to determine the stimulus reactivity. The sensitivity of the stabilimeter was routinely calibrated to ensure consistency between chambers and across sessions.

\section{Procedures}

In the demonstration of PPI of the acoustic startle reflex, subjects were presented with a series of discrete trials comprising a mixture of four types of trials. These included pulse-alone trials, prepulse-plus-pulse trials, prepulse-alone trials, and trials in which no discrete stimulus, other than the constant background noise, was presented. A reduction of startle magnitude in prepulse-plus-pulse trials relative to that in pulse-alone trials constitutes PPI. The pulse stimulus employed was $120 \mathrm{~dB}_{\mathrm{A}}$ in intensity and $40 \mathrm{~ms}$ in duration. Prepulses of various intensities were employed: $69,73,77$, and $81 \mathrm{~dB}_{\mathrm{A}}$, which corresponded to $4,8,12$, and $16 \mathrm{~dB}_{\mathrm{A}}$ above background, respectively. The duration of prepulse stimuli was $20 \mathrm{~ms}$. In Experiment 3, an additional level of prepulse $\left(85 \mathrm{~dB}_{\mathrm{A}}\right.$, ie $+20 \mathrm{~dB}_{\mathrm{A}}$ above background) was incorporated into the session definition, with the addition of the corresponding prepulse-alone and prepulse-pluspulse trials, in order to facilitate the detection of changes in prepulse reactivity. The stimulus onset asynchrony (SOA) of 
the prepulse and pulse stimuli on prepulse-plus-pulse trials was $100 \mathrm{~ms}$.

A session began with the animals being placed into the Plexiglas enclosure. They were acclimatized to the apparatus for $2 \mathrm{~min}$ before the first trial began. The first six trials consisted of startle-alone trials only, and they served to habituate and stabilize the animals' startle response. Subsequently, the animals were presented with 12 blocks of discrete test trials. Each block consisted of one trial of each of the following trial types: startle-alone, prepulseplus-pulse trials of each of the four (or five in Experiment 3) levels of prepulse, prepulse-alone of each of the four (or five in Experiment 3) levels of prepulse, and no stimulus (ie background alone). The interval between successive trials was variable with a mean of $15 \mathrm{~s}$ (ranging from 10 to $20 \mathrm{~s}$ ).

All procedures were conducted in accordance with the National Institutes of Health Guide for Care and Use of Laboratory Animals, and were approved by the Cantonal Veterinarian's Office of Zurich.

\section{Data Analysis}

The first six startle-alone trials were analyzed separately. The remaining trials were then subdivided into different trial types and the average values obtained for each individual animal. Three sets of data were then derived. The first set consisted of the reactivity scores obtained on prepulse-plus-pulse trials, and pulse-alone trials. A second set of data was calculated by converting the first data set into percentage score denoting the percent inhibition of startle response at each prepulse intensity by the formulae: (pulse-alone-prepulse-plus-pulse)/pulse-alone $\times 100 \%$. For each subject, the magnitude of PPI was calculated by the average of percent inhibition across the available range of prepulses in prepulse-plus-pulse trials. The third data set consisted of the reactivity scores obtained on prepulsealone trials, and on 'no-stimulus' trials. The 'no-stimulus' trials included in this data set served as a baseline condition against which the effect of increasing prepulse intensities could be evaluated. This directly paralleled the analysis of the first data set in which the pulse-alone condition was also included as a baseline condition against which the efficacy of prepulse to attenuate startle reaction to the pulse stimulus (ie PPI) could be assessed. These data were first subjected to natural logarithmic transformation to conform to the homogeneity and normality assumptions of parametric ANOVA. This was necessary because the prepulse reactivity scores were close to the lower boundary of the measuring scale, and the data distribution was therefore highly skewed. A summary of the untransformed prepulse reactivity values is presented in Table 1 for the reader's reference. The magnitude of prepulse reactivity for individual subjects was calculated by averaging the (lntransformed) reactivity scores across the available range of prepulses, including the 'no-stimulus' trials. All statistical analyses were carried out using the SPSS software implemented on a PC running the Windows XP operating system.

\section{RESULTS}

\section{Experiment 1: Effects of Apomorphine on PPI and Prepulse Reactivity}

In this experiment, 40 mice were allocated to the apomorphine treatment group (Apo), and 39 mice to the control group (VitC).

Prepulse inhibition. The disruptive effect of apomorphine upon PPI was evident in the analysis of the absolute level of startle reactivity (Figure 1a) and percent inhibition (Figure $1 \mathrm{~b}$ and inset). A $2 \times 5$ (treatment $\times$ prepulse levels) ANOVA of reactivity score obtained on pulse-alone and prepulse-plus-pulse trials yielded a significant effect of treatment $(\mathrm{F}=5.63, \mathrm{df}=1,77, p<0.05)$, prepulse levels $(\mathrm{F}=156.55, \mathrm{df}=4,308, p<0.001)$, and their interaction $(\mathrm{F}=3.62, \mathrm{df}=4,308, p<0.01)$. A priori pair-wise comparisons (as described by Winer, 1971) indicated that

Table I Summary of Untransformed Prepulse Reactivity Score

\begin{tabular}{|c|c|c|c|c|c|c|}
\hline & \multicolumn{6}{|c|}{ Intensities of the Prepulse stimulus ( $\mathrm{dB}_{\mathrm{A}}$ above the background of $65 \mathrm{~dB}_{\mathrm{A}}$ ) } \\
\hline & $+0 \mathrm{~dB}$ (no-strimulus) & $+4 \mathrm{~dB}$ & $+8 \mathrm{~dB}$ & $+12 \mathrm{~dB}$ & $+16 \mathrm{~dB}$ & $+20 \mathrm{~dB}$ \\
\hline Apomorphine & $4.57 \pm 0.31$ & $5.89 \pm 0.61$ & $11.23 \pm 1.41$ & $15.56 \pm 1.43$ & $22.91 \pm 2.49$ & - \\
\hline Vitamin C & $4.02 \pm 0.26$ & $4.18 \pm 0.32$ & $4.75 \pm 0.40$ & $7.27 \pm 0.78$ & $\mid 0.26 \pm 0.91$ & \\
\hline Amphetamine & $5.75 \pm 0.78$ & $6.22 \pm 1.05$ & $7.83 \pm 1.83$ & $12.30 \pm 2.76$ & $16.61 \pm 3.74$ & - \\
\hline $\mathrm{NaCl}$ & $3.07 \pm 0.26$ & $3.34 \pm 0.31$ & $3.42 \pm 0.34$ & $4.98 \pm 0.52$ & $9.00 \pm 1.43$ & \\
\hline \multicolumn{7}{|l|}{ Experiment 3} \\
\hline Vehicle+apomorphine & $5.13 \pm 0.76$ & $5.33 \pm 0.85$ & $10.44 \pm 1.56$ & $12.68 \pm 1.62$ & $|8.0| \pm 2.90$ & $21.99 \pm 5.17$ \\
\hline
\end{tabular}

The untransformed reactivity scores obtained on prepulse-alone trials in Experiments I-3 are summarized here. The reactivity scores (in arbitrary units) at each prepulse intensities, including the "no-stimulus", are presented as mean \pm SEM. 

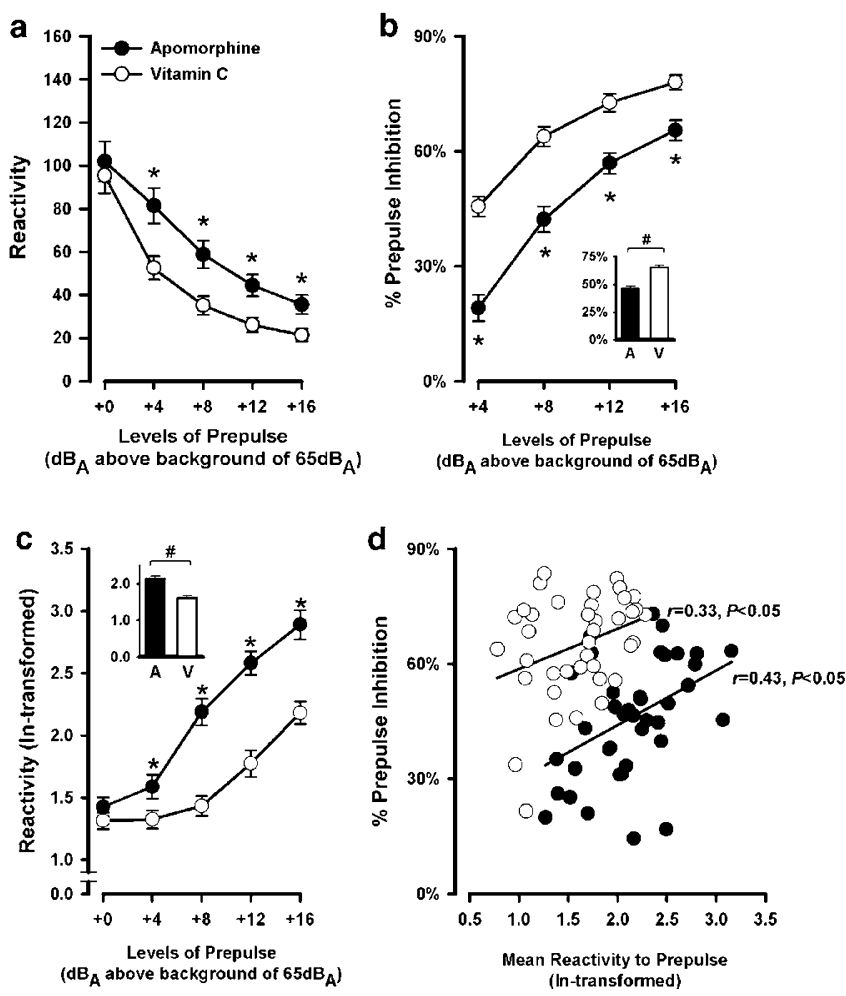

Figure I Results from Experiment I on the effects of systemic apomorphine on PPI and prepulse reactivity. (a) Mean reactivity scores obtained on pulse-alone and prepulse-plus-pulse trials. Reactivity in pulsealone trials is represented by the ' $+0 \mathrm{~dB}$ ' condition in this graph. (b) Mean percent inhibition in prepulse-plus-pulse trials. (c) Ln-transformed mean prepulse reactivity. Reactivity in the 'no-stimulus' trials is represented by the ' $+0 \mathrm{~dB}^{\prime}$ condition in this graph. (d) Scatter plot of average PPI against average prepulse reactivity on prepulse-alone (and no-stimulus) trials. Keys: - = apomorphine (A), $O=$ vitamin C vehicle control $(V)$. Error bars refer to \pm of the standard error of the mean value. Symbol * refers to the significant difference between the Apo and $\mathrm{VitC}$ groups at a given level of prepulse intensity ( $p<0.05$, two-tailed) as indicated by a priori comparison based on the pooled error variance of the overall ANOVA (Winer, 197I). Symbol \# in the inserted histogram in figures (b) and (c) refers to the significant main effect of treatment between apomorphine and vehicle injection.

apomorphine-treated animals showed a higher startle reactivity in prepulse-plus-pulse trials than vehicle controls $(p<0.05)$, but not on pulse-alone trials. A $2 \times 4$ (treatment $\times$ prepulse levels) ANOVA of percent inhibition confirmed the above impressions, yielding a main effect of prepulse levels $(\mathrm{F}=205.90, \mathrm{df}=3,231, p<0.001)$ and of treatment $(\mathrm{F}=34.39, \mathrm{df}=1,77, p<0.001)$ (Figure $1 \mathrm{~b})$.

Prepulse reactivity. Apomorphine did not affect the spontaneous reactivity obtained in trials in which no discrete stimulus other than the background noise was presented (ie ' $+0 \mathrm{~dB}_{\mathrm{A}}$ ' condition). Yet, the drug significantly enhanced the reactivity to all levels of prepulse above background as indicated by a priori pair-wise comparisons (all $p$ 's $<0.05$, see Figure 1c). These interpretations were confirmed by an ANOVA of the ln-transformed reactivity scores, which yielded a significant effect of treatment $(\mathrm{F}=28.39, \quad \mathrm{df}=1,77, \quad p<0.001), \quad$ prepulse $\quad(\mathrm{F}=105.97$, $\mathrm{df}=4,308, \quad p<0.001)$, as well as their interaction $(\mathrm{F}=11.18, \mathrm{df}=4,308, p<0.001)$.
Correlative analysis. A significant positive correlation between the mean (ln-transformed) prepulse reactivity and the mean percent PPI was separately obtained in the apomorphine $(r=+0.43, \mathrm{df}=38, p<0.01)$ and the vehicle groups $(r=+0.33, \mathrm{df}=37, p<0.05)$ (Figure 1d). Startle reactivity did not correlate significantly with the magnitude of PPI or spontaneous reactivity on no-stimulus trial. Prepulse reactivity and pulse reactivity also did not show any systemic relationship in the apomorphine group, but this attained statistical significance in the control group $(r=+0.37, \mathrm{df}=37, p<0.05)$.

Additional partial correlation analyses were conducted to examine if the association between prepulse reactivity and PPI magnitude observed in both groups could be accounted for by their separate covariance with startle reactivity. In both groups, partial correlation between prepulse reactivity and PPI magnitude remained highly significant with startle reactivity as the control variable (apomorphine: $r=+0.43$, $\mathrm{df}=37, p<0.01$; VitC: $r=+0.38, \mathrm{df}=36, p<0.05)$.

\section{Experiment 2: Effects of Amphetamine on PPI and Prepulse Reactivity}

In this experiment, 19 mice were allocated to the amphetamine treatment group (Amph), and 17 mice to the vehicle control group (Veh).

Prepulse inhibition. Analysis of startle reactivity indicated that amphetamine tended to enhance reactivity to the pulse stimulus in both pulse-alone and prepulse-plus-pulse trials (Figure 2a). This yielded a near-significant main effect of treatment $(\mathrm{F}=3.94, \mathrm{df}=1,34, p=0.055)$ in the analysis of the reactivity scores. PPI was evident in both groups, as indicated by reduced reactivity on prepulse-plus-pulse trials relative to pulse-alone trials. This led to a significant main effect of prepulse levels $(\mathrm{F}=107.16, \mathrm{df}=4,136, p<0.001)$, which did not interact with treatment $(\mathrm{F}<1.0)$. It can be readily discerned from Figure $2 \mathrm{a}$ that amphetamine elevated reactivity by a comparable magnitude in pulse-alone and prepulse-plus-pulse conditions. This may be taken as evidence that the prepulse was equally effective in inhibiting startle reactivity towards the impending pulse stimulus in both groups, that is, PPI was not disrupted by amphetamine. This conclusion is in conflict with the statistical analysis of percent inhibition across the four prepulse levels in prepulse-plus-pulse trials, which showed a clear disruption of PPI in the Amph group $(\mathrm{F}=11.37, \mathrm{df}=1,34$, $p<0.005)$. This was also accompanied by a significant main effect of prepulse levels $(\mathrm{F}=65.00, \mathrm{df}=3,102, p<0.001)$.

The two analyses thus yielded a conflicting conclusion as to whether PPI was attenuated or spared by amphetamine treatment. Similar interpretative problems of this kind are not uncommon in PPI literature involving various forms of treatment including drugs, lesions, and genetic manipulation (for a review, see Swerdlow et al, 2000). One view is that the significant effect obtained in the analysis of percent inhibition represents an artifact intrinsic to the calculation of percentages, such that an equivalent magnitude of reduction is translated into a smaller percentage reduction when there is an elevation of the baseline value. We examined this possibility by an analysis of covariance (ANCOVA) with mean \%PPI as the dependent variable and 
the mean reactivity on pulse-alone trials as the covariate. This yielded a significant effect of covariate $(\mathrm{F}=6.47$, $\mathrm{df}=1,33, p=0.016)$, while the main treatment effect remained highly significant $(\mathrm{F}=7.62, \mathrm{df}=1,33, p=0.009)$. This refutes the view that the significant reduction of PPI
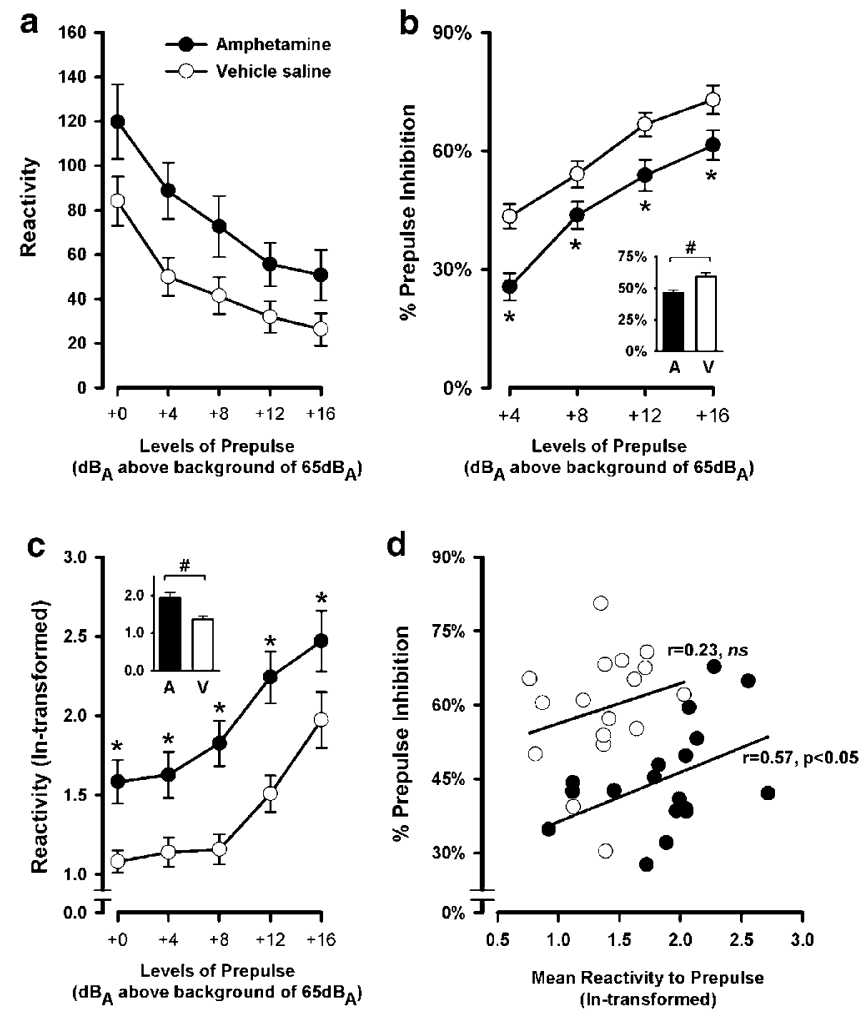

Figure 2 Results from Experiment 2 on the effects of systemic amphetamine on PPI and prepulse reactivity. (a) Mean reactivity scores obtained in pulse-alone and prepulse-plus-pulse trials. Reactivity in pulsealone trials is represented by the ' $+0 \mathrm{~dB}$ ' condition in this graph. (b) Mean percent inhibition on prepulse-plus-pulse trials. (c) Ln-transformed mean prepulse reactivity. Reactivity in the 'no-stimulus' trials is represented by the ' $+0 \mathrm{~dB}$ ' condition in this graph. (d) Scatter plot of average PPI against average prepulse reactivity in prepulse-alone (and no-stimulus) trials. Keys: - = amphetamine (A), $\mathrm{O}=0.9 \% \mathrm{NaCl}$ vehicle control $(\mathrm{NaCl}$ or $\mathrm{V}$ ). Error bars refer to \pm of the standard error of the mean value. Symbol * refers to the significant difference between the Amph and Veh groups at a given level of prepulse intensity $(p<0.05$, two-tailed) as indicated by a priori comparison based on the pooled error variance of the overall ANOVA (Winer, 197I). Symbol \# in the inserted histogram in figures (b) and (c) refers to the significant main effect of treatment between amphetamine and vehicle injection.

Figure 3 Results from Experiment 3 on the effects of haloperidol against apomorphine-induced disruption of PPI and enhancement of prepulse reactivity. (a) Mean reactivity scores obtained in pulse-alone and prepulseplus-pulse trials. Reactivity in pulse-alone trials is represented by the ' $+0 \mathrm{~dB}$ ' condition in this graph. (b) Mean percent inhibition on prepulseplus-pulse trials. (c) Ln-transformed mean prepulse reactivity. Reactivity in the 'no-stimulus' trials is represented by the ' $+0 \mathrm{~dB}$ ' condition in this graph. Keys: $\triangle=$ Hal $0.4 \mathrm{mg}+\mathrm{Apo}, \mathbf{\square}=$ Veh + Apo, $\mathrm{O}=$ Veh + VitC. Error bars refer to \pm of the standard error of the mean value. Symbol * refers to the significant difference against the $\mathrm{Veh}+\mathrm{VitC}$ group at a given level of prepulse intensity ( $p<0.05$, two-tailed) as indicated by a priori comparison based on the pooled error variance of the overall ANOVA (Winer, 197I). Symbol \# in the inserted histogram in figures (b) and (c) refers to the significant Newman-Keuls pair-wise comparison among treatment groups at $p<0.05$. (as expressed in percentages) by amphetamine could be completely explained in terms of the drug's effect in startle reactivity. This, however, does not suggest that covariance with startle reactivity did not contribute to the observed effect. The conclusion is that PPI as indexed by percent inhibition was significantly attenuated by amphetamine when the drug-induced elevation of startle reactivity was taken into account.
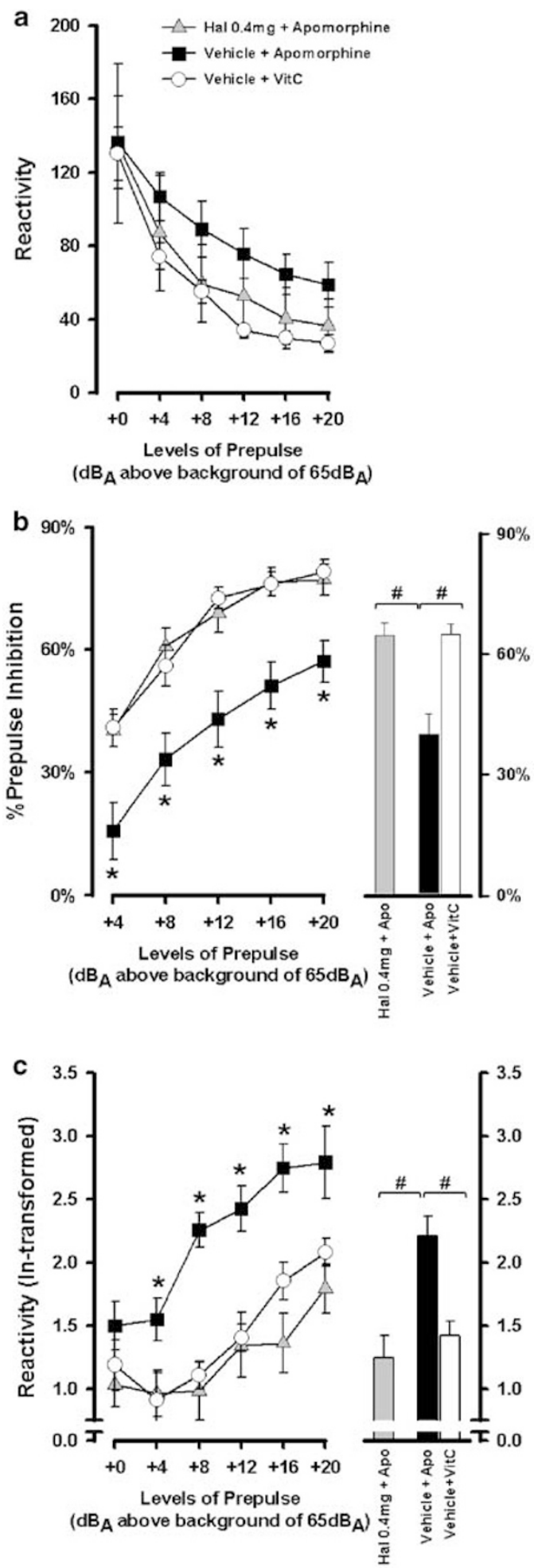
Prepulse reactivity. Amphetamine led to a general increase in the reactivity score obtained on prepulse-alone trials, with both groups showing higher reactivity towards prepulse of increasing intensity (Figure 2c). This effect was also apparent in trials with background noise only (' +0 ' condition of Figure 3c). These impressions were supported by the analysis of (ln-transformed) prepulse reactivity that yielded a significant effect of treatment $(\mathrm{F}=13.10, \mathrm{df}=1,33, \quad p<0.005)$ and of prepulse levels $(\mathrm{F}=32.74, \mathrm{df}=4,132, p<0.001)$, but not their interaction $(\mathrm{F}<1.0)$. The apparent elevation of prepulse reactivity therefore seemed to be motor-like, rather than sensory, in nature.

Correlative analysis. Although Experiment 2 was primarily designed to assess possible parallels with apomorphine in terms of the drug's effect on PPI and on prepulse reactivity, we present here a set of correlative analyses similar to Experiment 1 for the sake of consistency.

A significant correlation between the mean prepulse reactivity (ln-transformed score) and mean percent PPI was present in the Amph $(r=+0.57, \mathrm{df}=17, p<0.05)$, but not in the Veh group $(r=+0.23, \mathrm{df}=15, \mathrm{NS})$ (see Figure $2 \mathrm{~d}$ ). The significant correlation observed in the Amph groups remained significant in a partial correlation analysis while controlling for startle reactivity $(r=+0.59, \mathrm{df}=16$, $p<0.01)$. Spontaneous reactivity in no-stimulus trials also did not correlate with PPI magnitude in either group. The correlation between startle reactivity (ie in pulse-alone trials) and PPI magnitude was, however, significant in the Veh group ( $r=-0.64, p<0.01)$, but not in the Amph group. Startle reactivity and prepulse reactivity were not significantly related to each other in either group.

\section{Experiment 3: Effects of Haloperidol against Apomorphine-Induced Disruption of PPI and Enhancement of Prepulse Reactivity}

In this experiment, 10 mice were allocated to each of the following three treatment conditions: haloperidol + apomorphine ( $\mathrm{Hal}+\mathrm{Apo})$, vehicle + apomorphine (Veh + Apo), and vehicle + vehicle $(\mathrm{Veh}+\mathrm{VitC})$.

Prepulse inhibition. Inspection of startle reactivity scores (Figure 3a) and percent inhibition (Figure 3b) suggested that the presence of prepulse was less effective in inhibiting the startle magnitude to the pulse stimulus in the Veh + Apo group, replicating the results of Experiment 1. Haloperidol treatment nullified the disruptive effect of apomorphine on PPI, with performance between $\mathrm{Hal}+$ Apo and Veh $+\mathrm{VitC}$ groups being almost indistinguishable (Figure $3 \mathrm{~b}$ ). These impressions were supported by the analysis of percent inhibition, which revealed a significant main effect of treatment $(\mathrm{F}=13.64, \mathrm{df}=2,27, p<0.001)$ and of prepulse levels $(\mathrm{F}=73.80, \mathrm{df}=4,108, p<0.001)$. Newman-Keuls post hoc tests suggested that the Veh + Apo group exhibited a significant reduction in PPI $(p<0.05)$ relative to the other two groups that did not differ from each other. Analysis of startle reactivity scores (including pulse-alone and prepulse-plus-pulse trials) failed to reveal any significant difference among groups, and only yielded a significant effect of prepulse levels $(\mathrm{F}=47.14, \mathrm{df}=5,135, p<0.001)$.
Prepulse reactivity. Apomorphine led to a pronounced enhancement on prepulse reactivity as seen in Experiment 1 (Figure 3c). This effect was not associated with a significant increase in spontaneous reactivity (ie on no-stimulus trials). Haloperidol treatment completely antagonized this effect, as indicated by the response seen in the Hal-Apo group, which was not distinguishable from Veh $+\mathrm{VitC}$ controls. These impressions were supported by the main effect of treatment $(\mathrm{F}=11.50, \quad \mathrm{df}=2,27, \quad p<0.001), \quad$ of prepulse levels $(\mathrm{F}=33.87, \mathrm{df}=5,135, p<0.001)$, as well as their interaction $(\mathrm{F}=2.69, \mathrm{df}=10,135, p<0.01)$. Newman-Keuls post hoc comparisons revealed that the main effect of treatment was attributed solely to the enhanced prepulse reactivity in the $\mathrm{Veh}+$ Apo group compared to the other two groups.

\section{DISCUSSION}

The disruptive effects of apomorphine and amphetamine on PPI and the ability of haloperidol to reverse apomorphineinduced PPI disruption are well-established findings in rodent literature. The novel findings emerging from the present study concern the subjects' reactivity to the prepulse stimulus, and its relationship to the expression of PPI under the influence of a dopamine agonist. First, it was shown that an individual's prepulse reactivity was positively related to PPI magnitude (Experiment 1). Second, apomorphine enhanced the reaction to the prepulse stimulus, paralleling its disruptive effect on PPI (Experiment 1). Third, this effect of apomorphine on prepulse reactivity was antagonized by haloperidol, which was accompanied by the normalization of PPI performance at the same time (Experiment 3). These findings had until now escaped detection. Given the widespread use of PPI studies in schizophrenia and animal models of both this disease condition and its pharmacotherapy, these are important observations with potentially far-reaching consequences for both experimental control and theoretical interpretation.

Experiment 2 was included in the present study as an attempt to test whether the pattern of results obtained with apomorphine could be extended to the indirect dopamine agonist, amphetamine, because both drugs are commonly employed to produce disruption of PPI for the evaluation of potential antipsychotic drugs. The additional effects of amphetamine upon the reactivity on pulse-alone and nostimulus trials (see Figure 2a,c), however, have prevented a straightforward conclusion. As explained in the Results, there is, however, sufficient evidence to support the conclusion that the significant effect emerging from the percent inhibition analysis cannot be entirely dismissed as an artifact attributable to the drug's effect on startle reactivity. Unlike the effect of amphetamine on PPI, of which there are a number of demonstrations available for direct comparison, the effect of amphetamine on prepulse reactivity has not been reported before. Given that amphetamine's effect on no-stimulus trials is likely to be related to the drug's excitatory effect on locomotor activity, it would be premature to suggest that the apparent elevation of prepulse reactivity was similar in nature to that observed in Experiment 1 with apomorphine. One approach to overcome this confounding effect of amphetamine would require a detailed dose-response analysis. In contrast, the 
interpretation of the data derived from Experiments 1 and 3 did not suffer from similar caveats, because apomorphine affected neither startle reactivity nor spontaneous reactivity in no-stimulus trials. Furthermore, the former null effect cannot be attributed to a ceiling effect on startle, because amphetamine was effective in enhancing startle reactivity under precisely the same test conditions.

The lack of attention to prepulse processing or reactivity in the current literature may be partly attributed to the assumption that the prepulses are below the response threshold of startle response. However, the prepulses employed in most PPI experiments using the acoustic startle reflex paradigm are capable of eliciting reliable reactivity scores in mice (as confirmed by the present study) and in rats (Yee, 2000). Even though the magnitude of prepulse reactivity is substantially lower than the equivalent reading obtained in trials in which the startle-eliciting pulse stimulus is present, the prepulses can nonetheless lead to a measurable behavioral response ${ }^{1}$. Although both forms of reactions are measured using the same mechanistic device (through detection of whole body motion within a specified response window), reactivity to the prepulse stimulus and startle response to the pulse stimulus are likely to differ qualitatively, and the former should not merely be viewed as a form of 'miniature' startle. We have not attempted to pinpoint the precise nature of the prepulse reaction at present; instead, we have chosen to interpret it as a measure related to the detection and/or processing of the prepulse stimulus. This interpretation is supported by the observation that prepulse of increasing intensities led to correspondingly larger reactivity as observed here in all three experiments.

The presence of the prepulse stimulus, or more precisely, the subject's perception and processing of the prepulse, is essential to the demonstration of the PPI effect, and is therefore central to any theoretical account of PPI. Our results showed that subjects predisposed to react more strongly to the prepulse also tended to exhibit a higher level of PPI. This positive relationship was most clearly seen in Experiment 1, and was evident in both control and apomorphine-treated subjects. A similar relationship was also observed in Experiment 2, although the correlation only attained statistical significance in the amphetaminetreated animals but not in the control group, as the number of subjects was nearly half that of Experiment 1 . The latter was not surprising given the modesty of the effect size of this correlation-explaining $10-30 \%$ of the within-group variance. This positive relationship between prepulse reactivity and PPI magnitude is readily anticipated by the Graham (1975, 1980, 1992) hypothesis, and thereby provides some support for his psychological account of PPI, which predicts that enhanced processing of the prepulse stimulus can lead to reduced interference by the impending pulse stimulus, resulting thereby in stronger PPI. Graham's account asserts that the processing of

\footnotetext{
${ }^{1}$ We conducted additional runs of the present PPI protocol with four dead mice, and confirmed that none of these sessions generated consistent readings on prepulse-alone trials as seen in the three experiments reported here. This indicated that our measurements of prepulse reactivity did not stem from spurious vibrations associated with the delivery of the prepulse stimulus itself.
}

prepulse is causally related to enhanced PPI. However, the present data cannot determine the validity of such a causative claim. The observed positive correlation is also consistent with a sensory account based on individual variability in hearing acuity. It should be noted, however, that Graham's idea is still implicit in such an account.

Alternatively, the correlation between prepulse reactivity and PPI might stem from their separate covariance with a third variable. One candidate would be startle (or pulse) reactivity. To examine whether this could be a mediating variable solely responsible for the observed correlation, partial correlative analyses between prepulse reactivity and PPI were carried out (Chen and Popovich, 2002). In all three cases in which the correlation attained significance, the partial correlation remained highly significant. Thus, while variability in startle reactivity may have a role to play, it does not completely account for our observation. The interrelationships among prepulse reactivity, PPI, and startle reactivity clearly warrant further experimentation. Given that an inbred mouse strain was employed here, the variability studied here can be considered as largely of environmental and not genetic origin. It remains to be tested whether a similar pattern of results can be replicated in an out-bred strain that would more closely resemble the genetic heterogeneity in the human population.

The concomitant effects of apomorphine on prepulse reactivity (enhancement) and PPI (attenuation) demonstrated in Experiment 1 clearly do not conform to the observed positive relationship between prepulse reactivity and PPI. Generalization of the latter observation would lead one to expect that a treatment that is effective in enhancing prepulse reactivity should have potentiated PPI. Indeed, the suggestion by Davis et al (1990) that apomorphine disrupts PPI via reduced detectability of the prepulse is a logical extension of the same argument-reduction of prepulse detectability should lead to attenuation of PPI. It is clear that this cannot sufficiently account for the present observation in Experiment 1. This, however, does not exclude the possibility that other treatments do disrupt PPI via the mechanism postulated by Davis et al (1990). We suggest that apomorphine is likely to act via a separate mechanism, independent of the one offered by Davis et al (1990). This is because the positive correlation between prepulse reactivity and PPI was hardly affected by apomorphine, with the overall effect of apomorphine being best described as a shifting of this relationship as depicted in Figure 1d. Likewise, the efficacy of haloperidol in antagonizing both effects of apomorphine is to restore the initial shift induced by apomorphine (Figure $3 \mathrm{c}$ ).

We wish to emphasize that we are not suggesting that the dual effects of apomorphine observed here are causally related to one another, in the sense that one effect being directly responsible for the emergence of the other. Their conjoint appearance (Experiment 1) and conjoint normalization (Experiment 3) have led us to speculate that they might stem from a common psychological disturbance of a dopaminergic nature. One characterization is that apomorphine predisposes the subjects to attend to, or to process, stimuli that control subjects usually devote less attention to, or less processing resources to. That inattention exhibited by normal control subjects might be passive in nature, might result for instance from a low signal-to-noise ratio. It 
may also involve active inhibitory processes such as sensory-motor gating aimed at protecting the limitedcapacity processing downstream and to minimize potential interference.

Accordingly, the dual effects of dopamine agonists on PPI and prepulse reactivity can be understood as a form of enhanced distractibility. Enhanced distractibility may be achieved via a disruption of attentional control, and/or through enhanced signal-to-noise transmission. It has been suggested that enhanced dopamine transmission can improve the signal detection performance at the network level (Servan-Schreiber et al, 1990). This view offers a parsimonious account for the dual effects of dopamine agonists as well as of haloperidol reported here, and is consistent with impaired sustained attention and high distractibility commonly observed in schizophrenia patients (Kraeplin, 1919; Nuechterlein and Dawson, 1984). It remains to be ascertained whether the deficit in PPI reported in schizophrenic patients is also associated with an enhanced reactivity to the prepulse stimuli. If the PPI deficit in schizophrenia stems solely from a hyperdopaminergic state, our present data would predict that schizophrenia patients would also show enhanced reactivity to the prepulse stimulus.

Existing studies of PPI in animals and humans usually pay scant attention to the subjects' responses to the prepulse stimulus alone. In the limited studies available, there is evidence that prepulse detectability can influence PPI in humans in a direction similar to what we observed in mice (eg Norris and Blumenthal, 1996; Filion et al, 1993). A recent attempt in healthy human subjects, on the other hand, suggested that the expression of PPI was indistinguishable between trials in which the subjects expressed a conscious knowledge of the presence of the prepulse and trials in which they failed to do so (Postma et al, 2001). This seems to disagree with our present finding if conscious prepulse detection in human is equated with enhanced prepulse reactivity in mice. A number of key methodological differences that might be responsible for the divergent results between laboratories have been extensively discussed by Postma et al (2001). Here, we emphasize the most pertinent differences in the measurement of prepulse processing. Firstly, Postma et al (2001) obtained this measure through subjective report, which can be prone to subjective bias, and therefore may not faithfully reflect true detectability, especially as self-reports are typically obtained at the end of a trial, at a time when retroactive interference due to the highly salient pulse stimulus could have taken place. In our preparation, prepulse reactivity was measured in real-time, within a window of $65 \mathrm{~ms}$ from the onset of the prepulse stimulus. Admittedly, we measured prepulse reactivity on prepulse-alone trials instead of on prepulseplus-pulse trials due to technical limitation. Yet, this would not have mattered given that our response window was sufficiently short relative to the SOA ( $100 \mathrm{~ms})$ between prepulse and pulse in our prepulse-plus-pulse trials. Secondly, the correlative analysis that we conducted was focused on individual differences, whereas the Postma et al (2001) comparison was conducted within subjects. Hence, the possibility that the relationship between prepulse reactivity and PPI that we observed might only be reliably detected in the remaining population level. This clearly warrants further investigation in both humans and in animals.

Finally, the emphasis on prepulse reactivity analysis demonstrated here offers a new opportunity to re-examine a host of experimental manipulations that are known to affect the expression of PPI in animals (see Geyer et al, 2001), and in different patient populations (see Braff et al, 2001). For example, the attenuation of PPI following medial prefrontal cortex lesions was not associated with any changes in prepulse reactivity (Yee, 2000). The mechanism underlying this form of PPI disruption may therefore be distinct from that associated with systemic apomorphine treatment. It is expected that a renewed interest in prepulse processing would lead to a finer distinction between potentially different modes of PPI disruption that might be mediated by dissociable neural mechanisms.

\section{ACKNOWLEDGEMENTS}

This study was supported by the Swiss Federal Institute of Technology, Zurich. BK Yee was further supported by the National Center of Competence in Research (NCCR): Neural Plasticity and Repair, Swiss National Science Foundation. We gratefully acknowledge Dr Tobias Bast's efforts in critical thinking, and thank Alan Ipekian and Peter Schmid for their technical and editorial expertise. We also remain indebted to the anonymous reviewers for their constructive criticism in the revision of this article.

\section{REFERENCES}

Braff DL, Geyer MA, Swerdlow NR (2001). Human studies of prepulse inhibition of startle: normal subjects, patient groups, and pharmacological studies. Psychopharmacology 156: 234-258.

Braff DL, Grillon C, Geyer MA (1992). Gating and habituation of startle reflex in schizophrenic patients. Arch Gen Psychiatry 49: 206-215.

Castellanos FX, Fine EJ, Kaysen D, Marsh WL, Rapoport JL, Hallett M (1996). Sensorimotor gating in boys with Tourette's syndrome and ADHD: preliminary results. Biol Psychiatry 39: 33-41.

Chen PY, Popovich PM (2002). Correlation: Parametric and Nonparametric Measures. Sage Publications, Inc.: Newbury Park, CA.

Davis M, Mansbach RS, Swerdlow NR, Campeau S, Braff DL, Geyer MA (1990). Apomorphine disrupts the inhibition of acoustic startle induced by weak prepulses in rats. Psychopharmacology 102: $1-4$.

Filion DL, Dawson ME, Schell AM (1993). Modification of the acoustic startle-reflex eyeblink: a tool for investigating early and late attentional processes. Biol Psychol 35: 185-200.

Geyer MA, Krebs-Thomson K, Braff DL, Swerdlow NR (2001). Pharmacological studies of prepulse inhibition models of sensorimotor gating deficits in schizophrenia: a decade in review. Psychopharmacology 156: 117-154.

Geyer MA, McIlwain KL, Paylor R (2002). Mouse genetic models for prepulse inhibition: an early review. Mol Psychiatry 7: 1039-1053.

Graham FK (1975). The more or less startling effects of weak prestimulation. Psychophysiology 12: 238-248.

Graham FK (1980). Control of blink reflex excitability. In: Thompson RF, Hicks LH, Shvyrkov VB (eds). Neural Mechanisms of Goal-Directed Behavior and Learning. Academic Press: New York. pp 511-519.

Graham FK (1992). Attention: the heartbeat, the blink, and the brain. In: Campbell BA, Hayne H, Richardson R (eds). Attention 
and Information Processing in Infants and Adults: Perspectives from Human and Animal Research. Lawrence Erlbaum Associates: Hillsdale, NJ. pp 3-29.

Hoffman HS, Searle JR (1965). Acoustic variables in the modification of startle reaction in the rat. J Comp Exp Psychol 60: 53-58.

Kraeplin E (1919). Dementia Praecox and Paraphrenia (Translated by R.M. Barclay) Robert E. Krieger (1971): Huntington, NY.

Norris CM, Blumenthal TD (1996). A relationship between inhibition of the acoustic startle response and the protection of prepulse processing. Psychobiology 24: 160-168.

Nuechterlein KH, Dawson ME (1984). Information processing and attentional functioning in the developmental course of schizophrenic disorders. Schizophr Bull 10: 160-203.

Postma P, Kumari V, Hines M, Gray JA (2001). The relationship between prepulse detection and prepulse inhibition of the acoustic startle reflex. Psychophysiology 38: 377-382.

Servan-Schreiber D, Printz H, Cohen JD (1990). A network model of catecholamine effects: gain, signal-to-noise ratio, and behavior. Science 249: 892-895.

Snyder SH (1976). The dopamine hypothesis of schizophrenia: focus on the dopamine receptor. Am J Psychiatry 133: 197-202.

Swerdlow NR, Braff DL, Geyer MA (2000). Animal models of deficient sensorimotor gating: what we know, what we think we know, and what we hope to know soon. Behav Pharmacol 11: 185-204.

Swerdlow NR, Braff DL, Taaid N, Geyer MA (1994). Assessing the validity of an animal model of deficient sensorimotor gating in schizophrenic patients. Arch Gen Psychiatry 51: 139-154.

Swerdlow NR, Geyer MA (1998). Using an animal model of deficient sensorimotor gating to study the pathophysiology and new treatments of schizophrenia. Schizophr Bull 24: 285-301.

Swerdlow NR, Paulsen J, Braff DL, Butters N, Geyer MA, Swenson MR (1995). Impaired prepulse inhibition of acoustic and tactile startle response in patients with Huntington's disease. J Neurol Neurosurg Psychiatry 58: 192-200.

Winer BJ (1971). Statistical Principles in Experimental Design. McGraw-Hill Inc.: New York.

Yee BK (2000). Cytotoxic lesion of the medial prefrontal cortex abolishes the partial reinforcement extinction effect, attenuates prepulse inhibition of the acoustic startle reflex and induces transient hyperlocomotion, while sparing spontaneous object recognition memory in the rat. Neuroscience 95: 675-689.

Zorrilla EP (1997). Multiparous species present problems (and possibilities) to developmentalists. Dev Psychobiol 30: 141-150. 